\title{
Concerns, Healthcare Use, and Treatment Interruptions in Patients With Common Autoimmune Rheumatic Diseases During the COVID-19 Pandemic
}

\author{
Michael D. George $^{1}(\mathbb{D})$, Shilpa Venkatachalam ${ }^{2}(\mathbb{D})$, Shubhasree Banerjee ${ }^{1}(\mathbb{D})$ Joshua F. Baker $^{1}$ (D), \\ Peter A. Merkel ${ }^{1}$ (D), Kelly Gavigan², David Curtis², Maria I. Danila³, Jeffrey R. Curtis ${ }^{3}$, \\ and W. Benjamin Nowell ${ }^{2}$ (D)
}

\begin{abstract}
Objective. To assess concerns and healthcare-related behaviors of patients with autoimmune rheumatic diseases during the coronavirus disease 2019 (COVID-19) pandemic.

Methods. Adults from the United States with rheumatoid arthritis (RA), psoriatic arthritis (PsA), ankylosing spondylitis (AS), and systemic lupus erythematosus (SLE) from the ArthritisPower Patient-Powered Research Network and CreakyJoints patient community completed surveys. Concerns and behaviors were compared among patients with different autoimmune conditions, disease-modifying antirheumatic drug (DMARD) use, and geographic measures of urban status, income, education, and COVID-19 activity.

Results. Among 1517 participants (925 RA, 299 PsA, 185 AS, 108 SLE), mean age was 55.1 years, 88.3\% were female, and $89.5 \%$ were White. COVID-19 concerns were similar across the country and were higher in biologic users $(P<0.001)$. Avoidance of doctor's office visits $(56.6 \%)$ or laboratory testing $(42.3 \%)$ and use of telehealth $(29.5 \%)$ were more common in urban areas. Among participants receiving a DMARD without COVID-19 or other respiratory illness, $14.9 \%$ stopped a DMARD, with $78.7 \%$ of DMARD interruptions not recommended by a physician. DMARD stopping was more common in participants with lower socioeconomic status (SES) and in participants who avoided an office visit (OR 1.46, 95\% CI 1.04-2.04) or reported lack of telehealth availability OR 2.26 (95\% CI 1.25-4.08).

Conclusion. In the early months of the COVID-19 pandemic, patients with RA, PsA, AS, and SLE frequently avoided office visits and laboratory testing. DMARD interruptions commonly occurred without the advice of a physician and were associated with SES, office visits, and telehealth availability, highlighting the need for adequate healthcare access and attention to vulnerable populations during the pandemic.
\end{abstract}

Key Indexing Terms: antirheumatic drugs, COVID-19, health behavior, rheumatoid arthritis, spondyloarthritis, systemic lupus erythematosus

Coronavirus 2019 (COVID-19), caused by SARS-CoV-2 (severe acute respiratory syndrome coronavirus 2$)^{1,2}$, may be of particular concern to patients with autoimmune rheumatic diseases such as rheumatoid arthritis (RA), psoriatic arthritis
$M D G$ is supported by the National Institute of Arthritis and Musculoskeletal and Skin Diseases at the National Institutes of Health (K23 AR073931-01).

"The Arthritis and Rheumatic Disease COVID-19 Project" has received sponsorship support specifically from the Patient-Centered Outcomes Institute (PCORI), Eli Lilly and Company, and Janssen Pharmaceutical.

${ }^{1}$ M.D. George, MD, MSCE, Assistant Professor, S. Banerjee, MD, Assistant Professor, J.F. Baker, MD, MSCE, Assistant Professor, P.A. Merkel, MD, MPH, Professor, University of Pennsylvania, Philadelphia, Pennsylvania; ${ }^{2} S$. Venkatachalam, PhD, MPH, K. Gavigan, MPH, D. Curtis, BA, W.B. Nowell, PhD, MSW, Global Healthy Living Foundation, Upper Nyack, New York; ${ }^{3}$ M.I. Danila, MD, MSc, MSPH, Associate Professor, J.R. Curtis, MD, MS, MPH, Professor, University of Alabama at Birmingham, Birmingham, Alabama, USA.

J.R. Curtis and W.B. Nowell contributed equally to this work.

$M D G$ has received research support from Bristol-Myers Squibb for unrelated work. JB has received consulting fees from Bristol-Myers Squibb and Gilead. PM has received consulting fees from AbbVie, AstraZeneca, Biogen, Boeringher-Ingelheim, Bristol-Myers Squibb, Celgene, ChemoCentryx, CSL Behring, Forbius, Genentech/Roche, Genzyme/Sanofi, GlaxoSmithKline,

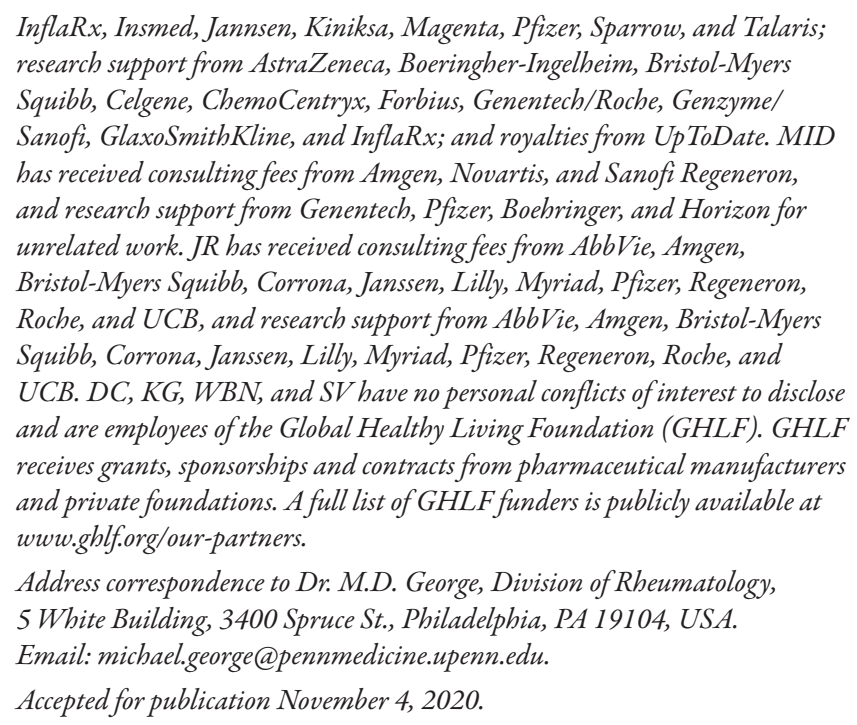
research support from AstraZeneca, Boeringher-Ingelheim, Bristol-Myers Squibb, Celgene, ChemoCentryx, Forbius, Genentech/Roche, Genzymel Sanofi, GlaxoSmithKline, and InflaRx; and royalties from UpToDate. MID has received consulting fees from Amgen, Novartis, and Sanofi Regeneron, and research support from Genentech, Pfizer, Boehringer, and Horizon for unrelated work. $J R$ has received consulting fees from AbbVie, Amgen, Bristol-Myers Squibb, Corrona, Janssen, Lilly, Myriad, Pfizer, Regeneron, Roche, and UCB, and research support from AbbVie, Amgen, Bristol-Myers Squibb, Corrona, Janssen, Lilly, Myriad, Pfizer, Regeneron, Roche, and UCB. DC, KG, WBN, and SV have no personal conflicts of interest to disclose and are employees of the Global Healthy Living Foundation (GHLF). GHLF receives grants, sponsorships and contracts from pharmaceutical manufacturers and private foundations. A full list of GHLF funders is publicly available at www.ghlf.org/our-partners.

Address correspondence to Dr. M.D. George, Division of Rheumatology, 5 White Building, 3400 Spruce St., Philadelphia, PA 19104, USA. Email:michael.george@pennmedicine.upenn.edu.

Accepted for publication November 4, 2020. 
(PsA), ankylosing spondylitis (AS), and systemic lupus erythematosus (SLE). These patients are at increased risk of infections, with immunosuppression and common comorbidities further affecting infection risk ${ }^{3,4}$.

To better understand how the COVID-19 pandemic has affected these patients, the ArthritisPower Patient-Powered Research Network (PPRN) registry (www.ArthritisPower. org $)^{5}$ launched a study to capture patient experiences during the pandemic across the United States, hypothesizing that patients with autoimmune diseases would have high levels of concern about COVID-19 and frequent disruptions in office visits, routine monitoring, and disease-modifying antirheumatic drug (DMARD) use that would vary geographically.

\section{MATERIALS AND METHODS}

Study population. Adults aged $>18$ years in the ArthritisPower PPRN or CreakyJoints patient community were sent email invitations. The ArthritisPower PPRN ${ }^{5,6}$ is a patient-led online registry of patients with inflammatory arthritis and other rheumatic conditions created as a joint venture of the patients and patient advocates of the Global Healthy Living Foundation, the CreakyJoints patient community, and researchers at the University of Alabama at Birmingham. Participants of partnering patient organizations were directed to a landing page (www.rheumcovid.com). Results are presented for surveys completed from March 29 to May 26, 2020, from participants in the US with a diagnosis of RA, PsA, SpA, or SLE. Participants provided consent and the protocol was approved by the Advarra Institutional Review Board (Protocol 00042873).

Data collection. Participants reported demographics, zip code, comorbidities, rheumatic conditions, and use of DMARD. For participants indicating multiple autoimmune conditions, a hierarchical approach was taken (SLE > PsA > AS > RA), similar to previous studies?

Participants were asked about their concerns about COVID-19 (5-point Likert scale), respiratory illnesses within the previous 2 weeks, COVID-19 testing/diagnoses, avoidance of office visits or laboratory/diagnostic testing, use and availability of telehealth ("telephone or telehealth visit"), and DMARD interruptions.

Geographic data. County rural versus urban status was defined using the National Center for Health Statistics classification ${ }^{8}$. Tertiles of COVID-19 activity in the patient's county relative to all other counties in the US on the survey date were defined using the cumulative cases per capita on that date, weighting counties by population 9 . Zip code-based median household income and education from the American Community Survey 2014-2018, five-year estimates were also divided into tertiles ${ }^{10}$.

Statistical analysis. Median scores for concerns about COVID-19 were graphed among states/territories with $\geq 5$ respondents. Kruskal-Wallis tests assessed for differences in concerns by geography ( 9 census divisions), autoimmune disease, biologic/Janus kinase (JAK) inhibitor use, urban versus rural geography, tertiles of zip code-based median household income, education, and county-based COVID-19 cases per capita.

The proportions of participants who had avoided an office visit, avoided testing, had a telehealth visit, or stopped medications were compared in the same subgroups of interest using univariate logistic regression to assess for statistical differences. DMARD stopping was assessed among participants reporting DMARD use, primarily evaluating patients without a respiratory illness or COVID-19 diagnosis to avoid participants who stopped DMARD because of illness.

\section{RESULTS}

Invitation emails were opened by 14,001 individuals, with 2895 clicking to access the study and 1935 completing the baseline
Table 1. Participant demographic and clinical characteristics.

$$
\mathrm{n}=1517
$$

\begin{tabular}{lc}
\hline Age, yrs & $55.1 \pm 11.7$ \\
Female & $1340(88.3)$ \\
White & $1358(89.5)$ \\
Hispanic & $75(4.9)$ \\
Rural $^{\mathrm{a}}$ & $214 / 1454(14.1)$ \\
Autoimmune conditions $^{\text {RA }}$ & \\
PsA & $925(61.0)$ \\
AS & $299(19.7)$ \\
SLE & $185(12.2)$ \\
Medications & $108(7.1)$ \\
bDMARD & \\
JAK inhibitor & $790(52.1)$ \\
Methotrexate & $158(10.4)$ \\
Hydroxychloroquine & $946(62.4)$ \\
Glucocorticoids & $537(35.4)$ \\
NSAID & $402(26.5)$ \\
Comorbidities & $373(24.6)$ \\
Hypertension & \\
Lung disease & $621(40.9)$ \\
DM & $518(34.2)$ \\
Heart disease & $179(11.8)$ \\
Kidney disease & $121(8.0)$ \\
Malignancy & $80(5.3)$ \\
Current smoking & $33(2.2)$ \\
PROMIS Anxiety, T-score & \\
\hline
\end{tabular}

Values are $\mathrm{n}(\%)$ or mean \pm SD. ${ }^{\text {a }}$ Rural status shown among participants with available zip code. ${ }^{\mathrm{b}}$ PROMIS Anxiety short form with range of 1-100, US adult population (mean $50 \pm$ SD 10). AS: ankylosing spondylitis; bDMARD: biologic disease-modifying antirheumatic drug; DM: diabetes mellitus; JAK: Janus kinase; NSAID: nonsteroidal antiinflammatory drug; PsA: psoriatic arthritis; PROMIS: Patient-Reported Outcomes Measurement Information System; RA: rheumatoid arthritis; SLE: systemic lupus erythematosus.

survey as of May 26, 2020. Excluding 20 participants outside the US and 398 without an autoimmune condition of interest left 1517 participants representing all 50 states and Puerto Rico (Figure 1). The mean age was 55.1, 88.3\% of participants were female, and $89.5 \%$ were White (Table 1 ).

A physician diagnosis of COVID-19 was reported by 11 participants $(0.7 \%)$, with $243(13.9 \%)$ reporting a respiratory illness without a diagnosis of COVID-19. Patients frequently wanted to be tested but were not, including $23.8 \%$ of patients who were not ill and $47.9 \%$ of patients reporting a respiratory illness. The most common barriers to testing were that the test was either not available (43.0\%) or not offered/recommended (36.8\%; Supplementary Table 1, available with the online version of this article).

Concerns about COVID-19. Overall, 698 (46.0\%) participants were extremely concerned and $515(34.0 \%)$ moderately concerned about COVID-19, and 803 (52.9\%) and $414(27.3 \%)$ noted that their autoimmune disease extremely or moderately affected their concerns about COVID-19, respectively 


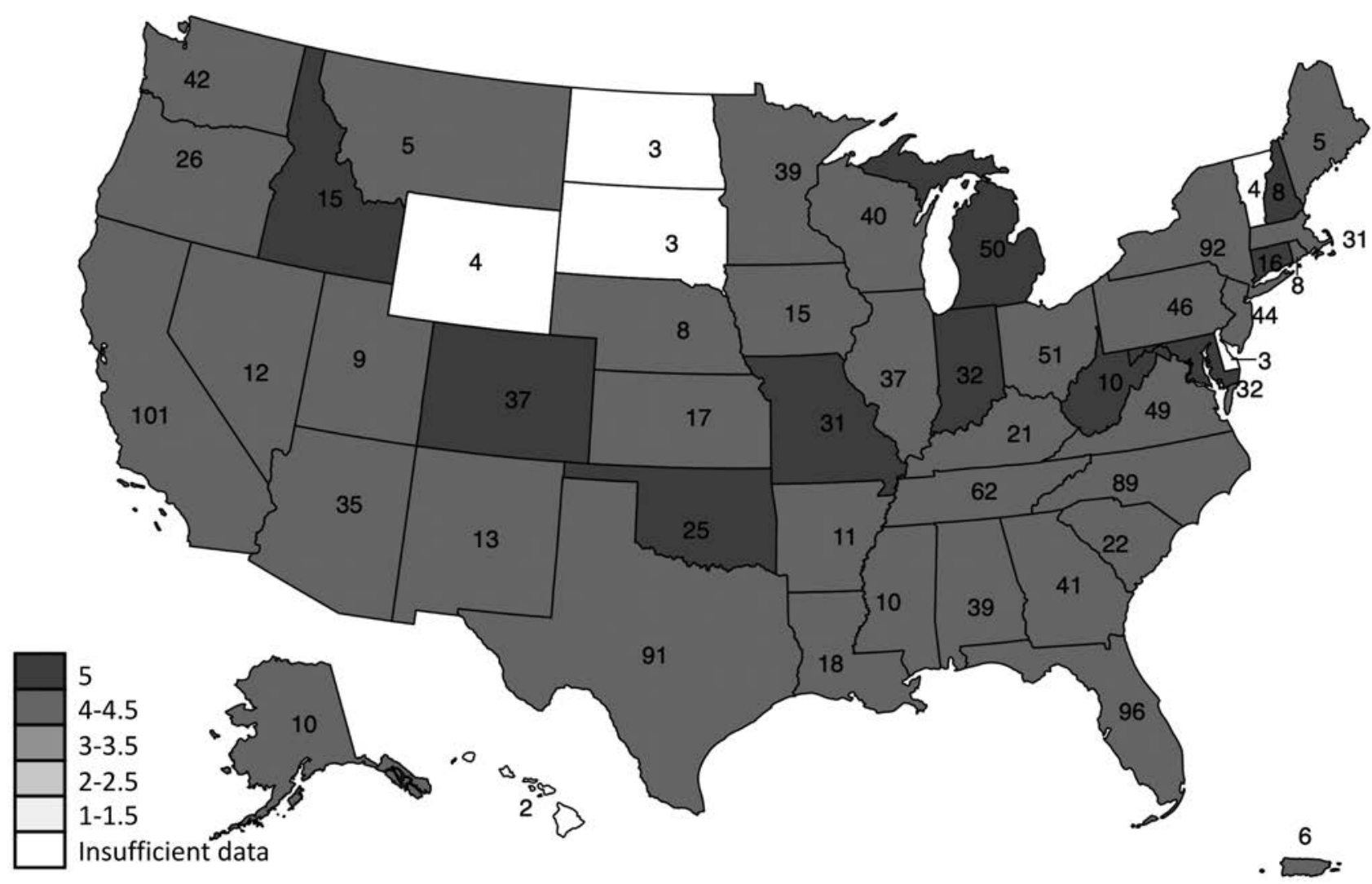

Figure 1. Location of participants and concerns about COVID-19. Number of participants by state/territory are shown. Shades represent median participant concern about COVID-19 in each state on a 5-point Likert scale, excluding states with < 5 participants. COVID-19: coronavirus 2019.

(Supplementary Figure 1, available with the online version of this article). Levels of concern were high across the US (Figure 1, Supplementary Figure 2), with no significant difference by geography, urban versus rural, median household income, or education level. Concerns were higher in participants receiving biologics/JAK inhibitors $(P<0.01$; Supplementary Figure 1$)$.

Effects on healthcare. Avoidance of doctor's office visits, laboratory testing, and other testing was reported by $56.6 \%, 42.3 \%$, and $36.0 \%$ of participants, respectively, with higher rates in participants with SLE (all $P<0.05$ ). Participants receiving a biologic/JAK inhibitor, those in urban or higher COVID-19 activity counties, and participants with greater COVID-19 concerns were more likely to avoid office visits or testing (Table 2; Supplementary Table 2, available with the online version of this article).

Telehealth visits were reported by 448 (29.5\%) participants, more commonly by participants in urban locations $30.7 \%$ vs $22.4 \%, P=0.02$; Table 2). Overall, 951 (62.7\%) participants reported that their doctor's office offered telehealth, 474 (31.2\%) did not know, and 92 (6.1\%) reported that telehealth was not available.

DMARD interruptions. Among participants treated with DMARD who did not report a respiratory illness or COVID-19 diagnosis, 169/1132 (14.9\%) stopped a DMARD. More frequent stopping was associated with biologic/JAK inhibitor use, lower education, lower or higher median household income, and greater COVID-19 concerns (Table 2; Supplementary Table 2, available with the online version of this article). Only $21.3 \%$ of these DMARD interruptions were recommended by a physician (Supplementary Table 3). Participants who avoided an office visit were more likely to stop DMARD [106/622 (17.0\%) vs 63/510 (12.4\%), OR 1.46 (95\% CI 1.04-2.04), $P=0.03$ ]. Telehealth use was not associated with stopping a DMARD (OR 1.04, 95\% CI 0.72-1.49), but DMARD stopping was more common among participants who reported that telehealth was not available $[17 / 67(25.4 \%)]$ versus those reporting telehealth availability [91/695 (13.1\%), OR 2.26 (95\% CI 1.25-4.08), $P<0.01]$, with even stronger associations among patients who avoided office visits [OR 3.01 (95\% CI 1.43-6.34), $P<0.01$; Supplementary Table 4].

DMARD interruptions were reported by $48 / 172$ (27.9\%) and $7 / 11(63.6 \%)$ patients reporting respiratory illness or COVID-19 diagnosis, respectively; $56.4 \%$ of these interruptions were recommended by a physician (Supplementary Table 3, available with the online version of this article).

Sources of information. The news media (84.4\%) and government websites $(67.4 \%)$ were the most common sources for information about COVID-19 (Supplementary Table 5, available with the online version of this article). Healthcare providers were more commonly a source of information among participants reporting a telehealth visit $(63.4 \%$ vs $34.9 \%$, $P<0.001)$. 
Table 2. Association of patient characteristics with healthcare utilization and medication interruptions.

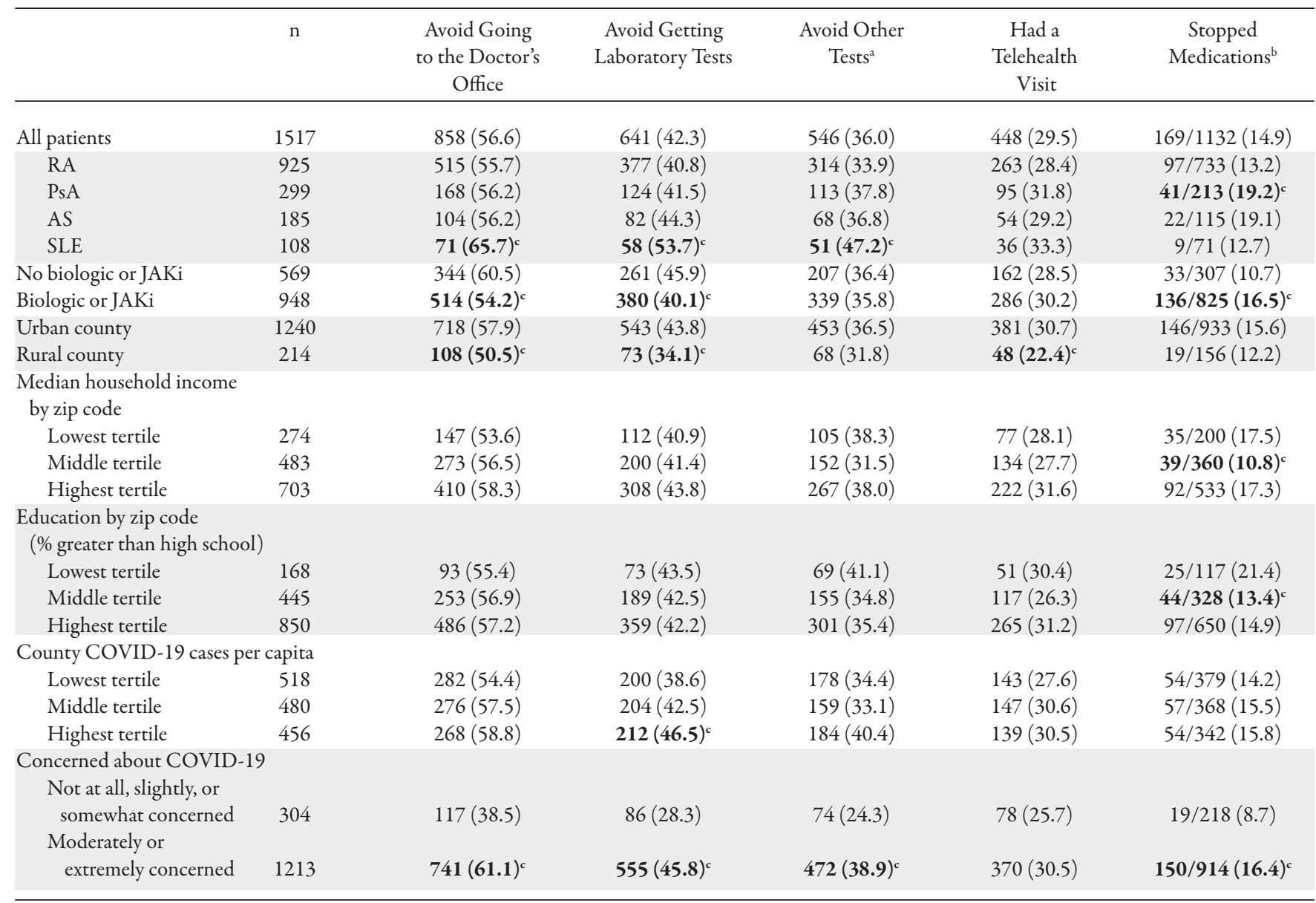

Proportions compared to the reference groups (RA, no biologics/JAKi, urban residence, lowest tertile of median household income, education, or COVID-19 cases per capita, and lower COVID-19 concern) using univariate logistic regression (OR and CI in Supplementary Table 2, available with the online version of this article). ${ }^{a}$ Other tests include radiographs. ${ }^{b}$ Proportion stopping medications is among patients who were receiving immunosuppression/immunomodulatory therapy and did not report a respiratory illness or diagnosis of COVID-19. ${ }^{\mathrm{c}} P<0.05$ versus the reference group. AS: ankylosing spondylitis; COVID-19: coronavirus disease 2019; JAKi: Janus kinase inhibitor; PsA: psoriatic arthritis; RA: rheumatoid arthritis; SLE: systemic lupus erythematosus.

\section{DISCUSSION}

This study of patients with common autoimmune rheumatic diseases across the US during the early months of the COVID-19 pandemic found that participants had high levels of concern about COVID-19 and frequently avoided office visits and testing. Of particular concern, approximately $15 \%$ had stopped one of their DMARD, especially patients who avoided office visits and did not have telehealth available.

Most medication interruptions in patients without illnesses occurred without a physician recommendation, similar to previous findings ${ }^{11}$. Although there are limited data on how immunosuppression affects the risk of severe COVID-19 $9^{12,13,14}$, medication interruptions can risk disease flares and glucocorticoid increases, both associated with infection risk ${ }^{15}$. The American College of Rheumatology recommends continuing DMARD unless patients are exposed or infected ${ }^{16}$, but these recommendations may not reach patients or be accepted by them. In this study, participants most frequently had obtained information about COVID-19 from news sources or government
Websites, which may not include information specific to patients with autoimmune disease. Participants who had avoided office visits or who reported that telehealth was not available were the most likely to have stopped a DMARD. Higher rates of stopping a DMARD among participants with lower socioeconomic status are concerning and require further investigation.

Participants in urban areas more frequently avoided office visits or testing for their autoimmune condition, and these areas were also those with the most telehealth use. These observations could be due to increased concerns or restrictions around clinic office visits, greater availability of or access to healthcare providers, or greater availability of telehealth technology/infrastructure in these areas. Although socioeconomic status was not associated with telehealth use, participants tended to be of higher socioeconomic status and might be more likely to be telehealth users; continued assessment of inequities in access to care and the optimal use of telehealth are important as the pandemic continues $^{17}$.

Several limitations are important to note. Patients who 
are survey respondents and members of online communities or networks may have different concerns and behaviors than the general population. Respondents were primarily White, and results may differ in racial/ethnic minority populations. Participants self-reported autoimmune and COVID-19 diagnoses, although they were specifically asked about physician diagnoses. We could not evaluate whether medication interruptions contributed to disease flares.

In conclusion, participants with autoimmune rheumatic disease across the US were highly concerned about COVID-19 and frequently avoided office visits or tests. Participants often stopped medications without the advice of a physician, especially those who had missed office visits and did not have telehealth access. Physicians should ask patients about medication concerns and interruptions, proactively contact patients who have missed visits, and ensure patients are aware of available options for accessing care. Continued assessment of disruptions in care, with a particular focus on vulnerable populations, is of critical importance as the pandemic continues.

\section{ACKNOWLEDGMENT}

We would like to recognize the partnering patient organizations that are disseminating this survey, including the Lupus Allied Disease Association (LADA), International Foundation for Autoimmune \& Autoinflammatory Arthritis (IFAA), American Bone Health, and Myositis Support and Understanding; Jennifer Walker and Kristine Carandang for reviewing and editing the survey; and the patients who have contributed and who continue to participate in this ongoing project.

\section{ONLINE SUPPLEMENT}

Supplementary material accompanies the online version of this article.

\section{REFERENCES}

1. Zhou P, Yang XL, Wang XG, Hu B, Zhang L, Zhang W, et al. A pneumonia outbreak associated with a new coronavirus of probable bat origin. Nature 2020;579:270-3.

2. Wu Z, McGoogan JM. Characteristics of and important lessons from the coronavirus disease 2019 (COVID-19) outbreak in China: summary of a report of 72314 cases from the Chinese Center for Disease Control and Prevention. JAMA 2020;323:1239-42.

3. Listing J, Gerhold K, Zink A. The risk of infections associated with rheumatoid arthritis, with its comorbidity and treatment. Rheumatol 2013;52:53-61.

4. Ogdie A, Maliha S, Shin D, Love TJ, Baker J, Jiang Y, et al. Cause-specific mortality in patients with psoriatic arthritis and rheumatoid arthritis. Rheumatol 2017;56:907-11.

5. Patient-Centered Outcomes Research Institute. ArthritisPower (ARthritis Partnership with Comparative Effectiveness
Researchers). [Internet. Accessed November 16, 2020.] Available from: www.pcori.org/research-results/2015/arthritispower-arthritispartnership-comparative-effectiveness-researchers

6. Nowell WB, Curtis D, Thai M, Wiedmeyer C, Gavigan K, Venkatachalam S, et al. Digital interventions to build a patient registry for rheumatology research. Rheum Dis Clin North Am 2019;45:173-86.

7. Ogdie A, Yu Y, Haynes K, Love TJ, Maliha S, Jiang Y, et al. Risk of major cardiovascular events in patients with psoriatic arthritis, psoriasis and rheumatoid arthritis: a population-based cohort study. Ann Rheum Dis 2015;74:326-32.

8. National Center for Health Statistics. Urban rural classification scheme for counties. [Internet. Accessed November 16, 2020.] Available from: www.cdc.gov/nchs/data_access/urban_rural.htm

9. USAFacts. US coronavirus cases and deaths. [Internet. Accessed November 16, 2020.] Available from: usafacts.org/visualizations/ coronavirus-covid-19-spread-map

10. Social Explorer. U.S. Census Bureau. American Community Survey 2014-2018 5-Year Estimates. [Internet. Accessed November 16, 2020.] Available from: www.socialexplorer.com/pub/reportdata/ HtmlResults.aspx ?reportid=R12543316

11. Michaud K, Wipfler K, Shaw Y, Simon TA, Cornish A, England $\mathrm{BR}$, et al. Experiences of patients with rheumatic diseases in the United States during early days of the COVID-19 pandemic. ACR Open Rheumatol 2020;2:335-43.

12. Haberman R, Axelrad J, Chen A, Castillo R, Yan D, Izmirly P, et al. COVID-19 in immune-mediated inflammatory diseases - case series from New York. N Engl J Med 2020;383:85-8.

13. Favalli EG, Ingegnoli F, De Lucia O, Cincinelli G, Cimaz R, Caporali R. COVID-19 infection and rheumatoid arthritis: faraway, so close! Autoimmun Rev 2020;19:102523.

14. Gianfrancesco M, Hyrich KL, Al-Adely S, Carmona L, Danila MI, Gossec L, et al; COVID-19 Global Rheumatology Alliance. Characteristics associated with hospitalisation for COVID-19 in people with rheumatic disease: data from the COVID-19 Global Rheumatology Alliance physician-reported registry. Ann Rheum Dis 2020;79:859-66.

15. Au K, Reed G, Curtis JR, Kremer JM, Greenberg JD, Strand V, et al; CORRONA Investigators. High disease activity is associated with an increased risk of infection in patients with rheumatoid arthritis. Ann Rheum Dis 2011;70:785-91.

16. Mikuls TR, Johnson SR, Fraenkel L, Arasaratnam RJ, Baden LR, Bermas BL, et al. American College of Rheumatology guidance for the management of adult patients with rheumatic disease during the COVID-19 pandemic. Arthritis Rheumatol 2020;72:1241-51.

17. Feldman $\mathrm{CH}$, Ramsey-Goldman R. Widening disparities among patients with rheumatic diseases in the COVID-19 era: an urgent call to action. Arthritis Rheumatol 2020;72:1409-11. 